\title{
L'acier en France en 2004
}

Extraits du rapport d'activité

de la Fédération Française de l'Acier

\section{EDITORIAL}

2004, dans un contexte de forte accélération de l'activité économique mondiale, a été une année record pour la sidérurgie mondiale avec une production globale qui, pour la première fois, a dépassé le milliard de tonnes.

Si l'ensemble des pays a connu une accélération de son rythme de croissance économique par rapport à 2003, c'est dans la zone asiatique et plus particulièrement en Chine, qu'elle a été la plus élevée, suivie de près par l'Amérique latine et les dix nouveaux pays membres de l'Union européenne, la zone euro, pour sa part, progressant plus faiblement.

La production d'acier a bénéficié, à des degrés divers, de cette forte croissance. Si la Chine a encore accentué sa progression $(+23 \%)$, il faut noter la bonne performance de l'Union européenne et notamment celle de la France, avec une augmentation de $5,1 \%$.

En 2004, la sidérurgie a poursuivi son mouvement de consolidation avec l'intégration de CST dans Arcelor et la création d'un groupe mondial, Mittal Steel.

La demande mondiale d'acier, tirée par la Chine, a continué à créer les tensions déjà observées en 2003, à savoir la montée des prix des matières premières, des tarifs du fret et, en conséquence, ceux de l'acier. La FFA a été interpellée par les représentants de secteurs utilisateurs tels la mécanique, l'automobile et le bâtiment, mécontents de la répercussion de ces hausses sur le prix de l'acier. Dans un souci d'écoute, d'information et de dialogue, la fédération a organisé plusieurs réunions avec les représentants des filières auto et bâtiment. Elle a également participé, à la demande du ministère de l'industrie, à deux tables rondes réunissant les participants et a travaillé, en concertation avec l'INSEE, à l'établissement d'un tableau de bord regroupant 47 indicateurs représentatifs de l'évolution des coûts.

Les sidérurgistes ont été fortement sollicités pour la préparation ou la mise en place de plusieurs projets de développement durable. En ce qui concerne le plan d'affectation des quotas d'émissions de $\mathrm{CO}_{2}$, les négociations menées avec les ministères ont été très serrées. Elles se sont traduites pour les aciéries françaises par un quota de 28,7 millions de tonnes par an pour la période 2005/2007. Ne pas utiliser plus de quota nécessitera des efforts significatifs d'amélioration d'efficacité énergétique. Le projet de nouvelle politique chimique européenne REACH a fortement mobilisé les adhérents via la FFA pour améliorer la proposition initiale de réglementation. Ces efforts seront poursuivis en 2005 en coopération avec les sidérurgistes européens.
Les performances de l'acier en 2004 s'apprécient aussi en termes de recherche et d'innovation avec notamment le projet européen ULCOS qui vise à réduire les émissions de $\mathrm{CO}_{2}$ pour la fabrication de l'acier, la mise au point de nouvelles solutions acier pour les moteurs de voitures ou encore de nouvelles nuances pour l'industrie automobile. Les qualités de ce matériau - sa durabilité et stabilité dans le temps, sa légèreté, sa mise en œuvre de haute technologie - auront également été illustrées par la réalisation du viaduc de Millau pour lequel près de 63000 tonnes d'acier ont été utilisées.

\section{Quelles perspectives pour 2005 ?}

La demande asiatique continuera à croître, mais à un rythme moins élevé ; celle de l'Union européenne pénalisée par l'atonie de la demande des ménages et la faiblesse des investissements, devrait connaître un taux de croissance moins soutenu qu'en 2004. Les prix des matières premières et de l'énergie resteront cependant structurellement élevés. Les sidérurgistes devront donc poursuivre leur politique d'écoute, de dialogue et de partenariat avec leurs clients pour faire face à cette évolution. Ils devront également saisir les opportunités des marchés en forte croissance pour constituer de nouvelles alliances. Ce mouvement de consolidation de la sidérurgie est nécessaire lorsque l'on constate que dans le secteur minier, les trois premiers producteurs de minerai de fer contrôlent $70 \%$ de la production mondiale alors que les dix premiers sidérurgistes représentent $30 \%$ de leur marché.

\section{口 LE MARCHE}

L'année 2004 a connu au niveau mondial une croissance économique de 4,9\%, constituant le rythme le plus élevé depuis 1988. La croissance, qui s'est accélérée en fin d'année 2003, s'est généralisée au cours du premier semestre de 2004, puis a perdu de son dynamisme au cours du second. Cette croissance a entraîné une forte accélération du commerce mondial et une envolée des prix des matières premières. Cette envolée ne s'est cependant que peu transmise dans l'inflation. Face à cette forte croissance, les créations d'emplois sont demeurées faibles, voire inexistantes en Europe.

La forte croissance des économies asiatiques avec en premier lieu celle de la Chine, mais également celle de l'économie américaine, s'est propagée à l'ensemble des zones dans le monde. La croissance de la Chine a été de 9,5\%, rythme voisin de celui de 2003. L'économie japonaise a connu également une forte croissance de ses exportations et un rebond de sa demande domestique. 
Croissance du produit intérieur brut (\%)

\begin{tabular}{|l|c|c|}
\hline & 2003 & 2004 \\
\hline Monde & 3,9 & 5,0 \\
\hline Union Européenne 25 & 1,1 & 2,3 \\
\hline Dont Union Européenne 15 & 0,9 & 2,0 \\
\hline Dont Union Européenne 10 & 3,1 & 5,3 \\
\hline Amérique du Nord & 2,8 & 4,3 \\
\hline Amérique du Sud & 2,2 & 6,2 \\
\hline Asie & 6,2 & 6,8 \\
\hline
\end{tabular}

Source : Rexecode

L'Amérique latine a enregistré de très bons résultats en 2004 grâce notamment à l'envolée des prix des matières premières, dont le pétrole et le minerai de fer. La croissance du Brésil a été vive atteignant $5,2 \%$ grâce aux très bons résultats de son commerce extérieur mais également à une reprise de la demande domestique. Le PIB de l'Argentine a progressé de 8,8\% et celui du Mexique de 4,2\%.

La croissance économique aux Etats-Unis a fait preuve d'une grande robustesse au cours de l'année 2004 atteignant 4,4\%. Elle s'est appuyée sur une croissance soutenue de la demande interne et des exportations.

L'Europe, en particulier la zone euro, a enregistré une croissance modeste au regard des autres zones économiques mondiales. L'absence de marge de manœuvre de la politique économique avec notamment la situation des déficits budgétaires, a pesé sur la croissance. L'augmentation des cours des matières premières et l'appréciation de l'euro ont réduit la compétitivité des entreprises européennes. La progression de ses exportations est restée très inférieure à celle de la demande mondiale. La demande interne n'a que peu évolué avec une hausse à peine supérieure à $1 \%$ sous l'effet d'une absence de reprise de l'emploi. Cependant, au sein de la zone, des disparités de situations ont été observées. En Allemagne, en Italie et aux Pays-Bas, l'activité est demeurée faible, voisine de 1,2\%. En Espagne, en Belgique et en France, elle a été supérieure à sa moyenne de longue période avec respectivement $2,7 \%, 2,6 \%$ et $2,3 \%$. Les exportations ont été le seul moteur de la croissance allemande tandis que les performances de la France à l'exportation ont été médiocres. En France, le moteur de la croissance économique a été le dynamisme de la dépense des ménages tant en consommation qu'en investissement.

La vigueur de la croissance du Royaume-Uni a de nouveau tranché avec celle des pays de la zone euro, avec une progression de 3,1\%. La forte augmentation des dépenses publiques et la demande soutenue des ménages ont été les moteurs de l'activité.

A nouveau, l'ensemble des dix nouveaux pays membres de l'Union a affiché de bonnes performances. La demande domestique a été dynamique tandis que cette zone a bénéficié de l'afflux d'investissements étrangers. La Pologne a largement consolidé sa reprise avec un PIB en croissance de 5,6\%.
Dans ce contexte, les secteurs utilisateurs d'acier de l'Union européenne ont enregistré un rebond voisin de 3\% en Europe des 15 tandis que celui des nouveaux pays membres avoisinait $8 \%$ de hausse.

Les immatriculations de voitures particulières ont sensiblement rebondi dans les pays de l'Europe des 15 avec une hausse de 2\%. Dans les nouveaux pays membres, les immatriculations de voitures neuves ont été en repli de 4,6\%. Les véhicules d'occasion importés ont fortement progressé dès l'adhésion de ces pays à l'Union européenne et se sont substitués aux ventes de véhicules neufs. En France, le marché a été stable après la chute sévère de $6,3 \%$ en 2003. La production de véhicules (voitures particulières et véhicules industriels) de l'Union européenne a progressé de $3 \%$ en 2004, a été stable dans les pays de l'UE à 15 et a progressé de $16 \%$ dans les dix nouveaux pays membres. En France, la production de voitures particulières a également été proche du niveau de 2003 tandis que celle de véhicules utilitaires légers et de véhicules industriels a fortement rebondi avec une croissance de $10 \%$. Au total, la production française de véhicules a augmenté de 1,3\%.

La production des industries mécaniques a nettement rebondi après deux années en repli. La croissance du secteur a atteint près de $4 \%$ dans les pays de l'UE à 15 , tirée par la forte hausse des exportations vers la zone asiatique avec notamment la Chine. Cette accélération des exportations a été très élevée en Allemagne. La demande domestique est demeurée faible dans la plupart des pays de la zone. Dans les dix nouveaux membres de l'UE, l'activité du secteur a également été soutenue, avec une croissance supérieure à $9 \%$. La France a bénéficié du rebond de la demande mondiale et l'activité a été en progression de 4,2\%. Parallèlement, le secteur du travail des métaux a progressé de près de $2,5 \%$ en UE 15 et de $17 \%$ dans les dix nouveaux membres de l'UE. L'activité de ce secteur en France est restée inférieure à la moyenne européenne, ne progressant que de $1,3 \%$.

Le secteur de la construction a poursuivi une évolution contrastée. La partie résidentielle a bénéficié de taux d'intérêts faibles et l'activité s'est maintenue à un niveau satisfaisant dans plusieurs pays européens, notamment en France. A l'opposé, le secteur non résidentiel privé a continué à souffrir de la faiblesse des investissements et a été en repli. La situation a été très différenciée selon les pays, l'activité a baissé de $4 \%$ en Allemagne tandis qu'elle a progressé au RoyaumeUni, en Espagne et en France. En France, l'activité de l'ensemble du secteur a augmenté de $3 \%$ environ. Les travaux dans le logement neuf et dans les bâtiments administratifs neufs ont fortement progressé avec des hausses proches de $10 \%$. A l'opposé, les travaux dans les bâtiments d'entreprises neufs ont reculé de $3 \%$. L'activité des travaux publics en France a été élevée avec une progression de 3\%.

Dans ce contexte, la consommation réelle d'acier a renoué avec la croissance en Europe et a été en hausse de $2 \%$ en France. L'accélération de l'activité économique a conduit à une hausse des stocks au cours de l'année dans l'ensemble des pays d'Europe. La consommation apparente d'acier en Europe des 15 a progressé de près de $4 \%$. Dans les nouveaux 
pays membres de l'UE, le dynamisme des secteurs consommateurs d'acier a entraîné une croissance de la consommation apparente d'acier de 10\%. En France, la consommation apparente d'acier a progressé de $6,7 \%$ (y compris demi-produits), de $5,2 \%$ pour les produits longs et $6,5 \%$ pour les produits plats.

L'approvisionnement du marché français en produits finis sidérurgiques a progressé de $6,6 \%,(+5 \%$ pour les livraisons en provenance des usines françaises et $+7,9 \%$ pour les importations).

\section{Le commerce extérieur de l'Union européenne à 25}

Les importations de produits sidérurgiques de l'UE à 25 en provenance des pays tiers ont augmenté de $1 \%$ (demi-produits exclus), de $3,1 \%$ pour les produits plats mais baissé de $3,3 \%$ pour les produits longs. Les importations en provenance de Russie et d'Ukraine ont poursuivi leur hausse avec respectivement $8,5 \%$ et $46 \%$. Les importations en provenance du sud-est asiatique ont enregistré une croissance moyenne de $15 \%$.

Les exportations de produits sidérurgiques vers les pays tiers ont enregistré une progression de près de $4 \%$ (hors demi-produits) mais avec des évolutions contrastées selon les produits : une baisse de $0,6 \%$ pour les produits plats et une hausse supérieure à $12 \%$ pour les produits longs. Les exportations vers l'Asie du Sud-Est se sont infléchies de 21\%, dont une chute de $39 \%$ vers la Chine. Les exportations vers les Etats-Unis se sont redressées et ont augmenté de $45 \%$ après le repli important de ces dernières années.

\section{Le commerce extérieur français}

Les importations françaises de produits sidérurgiques et de produits issus de la première transformation de l'acier ont augmenté de $9,9 \%$.
Les exportations françaises de produits sidérurgiques et de produits issus de la première transformation de l'acier ont progressé moins vite $(6,8 \%)$. Les exportations hors Europe ont évolué de façon différenciée selon les produits avec une baisse de $2 \%$ pour les produits sidérurgiques et une forte hausse $(26 \%)$ pour les produits de la première transformation. En résultante, le solde des échanges extérieurs de produits sidérurgiques et de produits de la première transformation s'est détérioré.

\section{- PRODUCTION MONDIALE D'ACIER}

La production mondiale d'acier a dépassé en 2004 le milliard de tonnes produites, atteignant 1057 millions de tonnes d'acier brut selon les estimations de l'IISI, correspondant à une croissance de $8,8 \%$ sur l'année.

Bien que cette hausse reflète des rythmes d'évolutions différents entre les différentes zones économiques mondiales, une hausse générale a été observée.

La production de l'Union européenne a été en hausse de $4,9 \%$, la France s'inscrivant légèrement au-dessus de la moyenne européenne, avec une croissance de $5,1 \%$. Le poids de l'Europe à 25 dans la production mondiale est de $18 \%$.

La production d'acier de la Turquie s'est accélérée de 11,9\% Les pays de la CEI ont enregistré une croissance plus modeste avec $4 \%$

La hausse a été à nouveau concentrée dans la zone asiatique qui a enregistré une croissance de $13,1 \%$. La production d'acier de la zone asiatique représente près de la moitié de la production mondiale avec $47 \%$. Ce dynamisme de la zone asiatique doit à nouveau beaucoup à la croissance de la production chinoise dont la hausse a été de $23 \%$. La production de la Chine a progressé à un rythme supérieur à $20 \%$ par an depuis l'année 2000 doublant son niveau depuis cette date. Au Japon, la hausse de la production d'acier a été limitée à $2 \%$.

En Amérique du Nord, la production a rebondi avec une hausse de 5,6\%, les Etats-Unis se situant dans cette moyenne.

\begin{tabular}{|c|c|c|c|c|c|c|c|c|c|}
\hline \multicolumn{10}{|c|}{ Commerce extérieur français (en 1000t) } \\
\hline & & \multicolumn{4}{|c|}{ Produits sidérurgiques* } & \multicolumn{4}{|c|}{ Produits de la première transformation } \\
\hline & & \multicolumn{2}{|c|}{$\begin{array}{l}\text { Exportations } \\
2004 \text { Var.\% }\end{array}$} & \multicolumn{2}{|c|}{$\begin{array}{l}\text { Importations } \\
2004 \text { Var. \% }\end{array}$} & \multicolumn{2}{|c|}{$\begin{array}{l}\text { Exportations } \\
2004 \text { Var. \% }\end{array}$} & \multicolumn{2}{|c|}{$\begin{array}{l}\text { Importations } \\
2004 \text { Var. \% }\end{array}$} \\
\hline \multicolumn{2}{|c|}{ Pays de l'Union européenne (24) } & 13922 & 7,9 & 12879 & 9,3 & 1633 & 0,8 & 2547 & 9,3 \\
\hline \multicolumn{2}{|c|}{ Pays tiers } & 2028 & $-2,1$ & 609 & 22,8 & 847 & 26,0 & 239 & 21,9 \\
\hline \multirow[t]{5}{*}{ dont: } & -Europe Occidentale & 559 & 14,8 & 104 & 10,6 & 136 & 4,6 & 69 & 38,0 \\
\hline & -Europe Centrale et Orientale & 17 & 6,3 & 94 & $-1,1$ & 34 & 78,9 & 90 & 11,1 \\
\hline & -Etats Unis & 419 & 30,5 & 20 & $-42,9$ & 109 & 34,6 & 9 & 28,6 \\
\hline & -Chine & 77 & $-62,8$ & 0 & & 44 & 100 & 4 & 300 \\
\hline & -Autres pays tiers & 956 & $-8,1$ & 391 & 43,8 & 524 & 24,8 & 67 & 17,5 \\
\hline \multicolumn{2}{|l|}{ Total } & 15950 & 6,5 & 13487 & 9,9 & 2480 & 8,2 & 2786 & 10,3 \\
\hline
\end{tabular}

Source: Douanes. (*) Produits sidérurgiques = produits finis sidérurgiques + demi-produits pour relaminage 


\begin{tabular}{|c|c|c|c|c|c|c|}
\hline & & \multicolumn{3}{|c|}{ Production (kt) } & \multicolumn{2}{|c|}{ Variations } \\
\hline & & 2002 & 2003 & 2004 & 03/02 & $04 / 03$ \\
\hline \multicolumn{2}{|c|}{ Union Européenne } & 180895 & 184209 & 193323 & $+1,8 \%$ & $+4,9 \%$ \\
\hline \multirow{20}{*}{ dont: } & Allemagne & 45015 & 44809 & 46374 & $-0,5 \%$ & $+3,5 \%$ \\
\hline & \multirow{2}{*}{$\begin{array}{l}\text { Autriche } \\
\text { Belgique }\end{array}$} & 6189 & 6261 & 6530 & $+1,2 \%$ & $+4,3 \%$ \\
\hline & & 11343 & 11135 & 11733 & $-1,8 \%$ & $+5,4 \%$ \\
\hline & Danemark & 392 & 0 & 0 & $-100,0 \%$ & $+0,0 \%$ \\
\hline & Espagne & 16408 & 16472 & 17684 & $+0,4 \%$ & $+7,4 \%$ \\
\hline & Finlande & 4003 & 4766 & 4831 & $+19,1 \%$ & $+1,4 \%$ \\
\hline & France & 20258 & 19758 & 20770 & $-2,5 \%$ & $+5,1 \%$ \\
\hline & Grèce & 1835 & 1701 & 1967 & $-7,3 \%$ & $+15,6 \%$ \\
\hline & Italie & 26066 & 26832 & 28414 & $+2,9 \%$ & $+5,9 \%$ \\
\hline & Luxembourg & 2719 & 2675 & 2684 & $-1,6 \%$ & $+0,3 \%$ \\
\hline & Pays-Bas & 6117 & 6571 & 6848 & $+7,4 \%$ & $+4,2 \%$ \\
\hline & Portugal & 920 & 730 & 720 & $-20,7 \%$ & $-1,4 \%$ \\
\hline & Royaume Uni & 11667 & 13268 & 13666 & $+13,7 \%$ & $+3,0 \%$ \\
\hline & Suède & 5754 & 5707 & 5978 & $-0,8 \%$ & $+4,7 \%$ \\
\hline & Etats Baltes & 521 & 521 & 521 & $+0,0 \%$ & $+0,0 \%$ \\
\hline & Hongrie & 2052 & 1984 & 1958 & $-3,3 \%$ & $-1,3 \%$ \\
\hline & Pologne & 8368 & 9107 & 10592 & $+8,8 \%$ & $+16,3 \%$ \\
\hline & République Tchèque & 6512 & 6783 & 7033 & $+4,2 \%$ & $+3,7 \%$ \\
\hline & Slovaquie & 4275 & 4588 & 4454 & $+7,3 \%$ & $-2,9 \%$ \\
\hline & Slovénie & 481 & 541 & 566 & $+12,5 \%$ & $+4,6 \%$ \\
\hline Autres Pay & s d'Europe de l'Ouest & 19120 & 21138 & 23859 & $+10,6 \%$ & $+12,9 \%$ \\
\hline dont : & Turquie & 16467 & 18298 & 20478 & $+11,1 \%$ & $+11,9 \%$ \\
\hline Bulgarie et & Roumanie & 7351 & 8008 & 8392 & $+8,9 \%$ & $+4,8 \%$ \\
\hline dont : & Bulgarie & 1860 & 2317 & 2350 & $+24,6 \%$ & $+1,4 \%$ \\
\hline & Roumanie & 5491 & 5691 & 6042 & $+3,6 \%$ & $+6,2 \%$ \\
\hline CEI & & 101089 & 106220 & 113112 & $+5,1 \%$ & $+6,5 \%$ \\
\hline dont : & Kazakhstan & 4814 & 4898 & 5385 & $+1,7 \%$ & $+9,9 \%$ \\
\hline & Russie & 59777 & 61450 & 65583 & $+2,8 \%$ & $+6,7 \%$ \\
\hline & Ukraine & 34050 & 36932 & 38738 & $+8,5 \%$ & $+4,9 \%$ \\
\hline Amérique & du Nord & 122949 & 126159 & 133255 & $+2,6 \%$ & $+5,6 \%$ \\
\hline dont : & Canada & 16002 & 15927 & 16281 & $-0,5 \%$ & $+2,2 \%$ \\
\hline & Etats Unis & 91587 & 93677 & 98946 & $+2,3 \%$ & $+5,6 \%$ \\
\hline & Mexique & 14010 & 15159 & 16730 & $+8,2 \%$ & $+10,4 \%$ \\
\hline Amérique & du Sud & 40858 & 43036 & 45871 & $+5,3 \%$ & $+6,6 \%$ \\
\hline dont : & Argentine & 4356 & 5033 & 5125 & $+15,5 \%$ & $+1,8 \%$ \\
\hline & Brésil & 29604 & 31147 & 32913 & $+5,2 \%$ & $+5,7 \%$ \\
\hline & Venezuela & 4164 & 3930 & 4561 & $-5,6 \%$ & $+16,1 \%$ \\
\hline Asie & & 394929 & 441166 & 499539 & $+11,7 \%$ & $+13,2 \%$ \\
\hline dont : & Chine Populaire & 182249 & 221165 & 272456 & $+21,4 \%$ & $+23,2 \%$ \\
\hline & Corée du Sud & 45390 & 46310 & 47521 & $+2,0 \%$ & $+2,6 \%$ \\
\hline & Inde & 28814 & 31779 & 32626 & $+10,3 \%$ & $+2,7 \%$ \\
\hline & Japon & 107745 & 110511 & 112716 & $+2,6 \%$ & $+2,0 \%$ \\
\hline & Taiwan & 18230 & 18832 & 19570 & $+3,3 \%$ & $+3,9 \%$ \\
\hline Moyen Ori & ent & 12492 & 13443 & 14253 & $+7,6 \%$ & $+6,0 \%$ \\
\hline Afrique & & 15808 & 16290 & 16702 & $+3,0 \%$ & $+2,5 \%$ \\
\hline dont : & Afrique du Sud & 9095 & 9481 & 9500 & $+4,2 \%$ & $+0,2 \%$ \\
\hline Australie-I & Jouvelle Zelande & 8292 & 8397 & 8282 & $+1,3 \%$ & $-1,4 \%$ \\
\hline Monde & & 903783 & 968066 & 1056588 & $+7,1 \%$ & $+9,1 \%$ \\
\hline
\end{tabular}


Production d'acier brut - indice 100 en 1950.

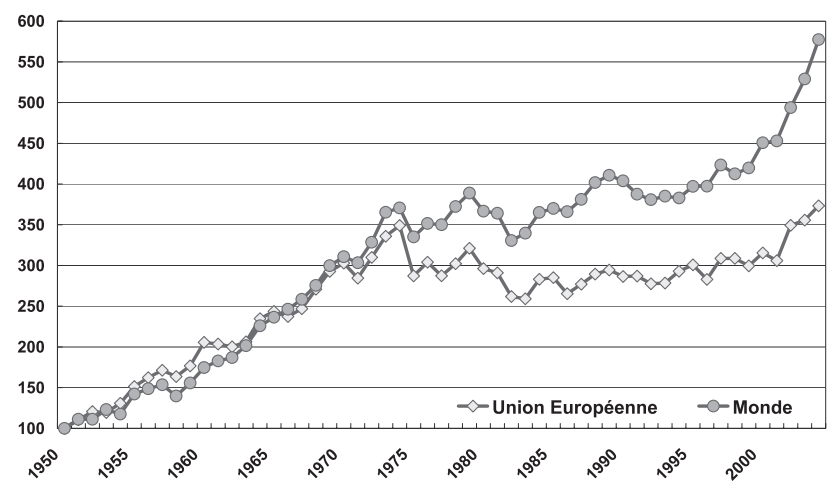

La production d'acier de l'Amérique du Sud a poursuivi une trajectoire positive avec une hausse de $6,6 \%$, qui fait suite à plusieurs années de croissances élevées au voisinage de $6 \%$ de hausse par an depuis 1999.

\section{L'ACIER EN FRANCE}

\section{Approvisionnements-Énergie}

\begin{tabular}{|l|c|c|c|c|}
\hline & \multicolumn{3}{|c|}{ Minerai de fer } \\
\hline & 2003 & 2004 & $\begin{array}{c}\text { Variation en } \% \\
2003 / 2002\end{array}$ \\
\hline $\begin{array}{l}\text { Importations } \\
\text { (milliers de tonnes) }\end{array}$ & 19936 & 21404 & \multicolumn{2}{|c|}{$+7,4 \%$} \\
\hline & Brésil & Australie & Mauritanie & Canada \\
\cline { 2 - 5 } $\begin{array}{l}\text { Principales sources } \\
\text { d'approvisionnement } \\
\text { à l'importation }\end{array}$ & $63,5 \%$ & $16 \%$ & $12,3 \%$ & $5 \%$ \\
\hline
\end{tabular}

\begin{tabular}{|l|c|c|c|}
\hline \multicolumn{4}{|c|}{ Ferrailles (milliers de tonnes) } \\
\hline & 2003 & $2004^{*}$ & Variation en \% \\
\hline Approvisionnements totaux & & & \\
\hline Collecte & 12012 & 12728 & $5,96 \%$ \\
\hline Importations & 3030 & 3462 & $14,26 \%$ \\
\hline Exportations & 5016 & 5598 & $11,60 \%$ \\
\hline Consommation & 10164 & 10601 & $4,30 \%$ \\
\hline
\end{tabular}

* chiffres provisoires

\begin{tabular}{|l|}
\hline \multicolumn{1}{|c|}{ Energie } \\
\hline Consommation de gaz naturel en hausse de $9,8 \%$ \\
\hline Consommation d'électricité en hausse de 1,6 \% \\
\hline Consommation de gaz sidérurgiques en hausse de 7,8 \% \\
\hline $\begin{array}{l}\text { Consommation de coke et de houille en hausse de 1,7 \% } \\
\text { et hausse de 5,3 \% des achats extérieurs }\end{array}$ \\
\hline
\end{tabular}

Production mondiale d'acier brut-année 2004.

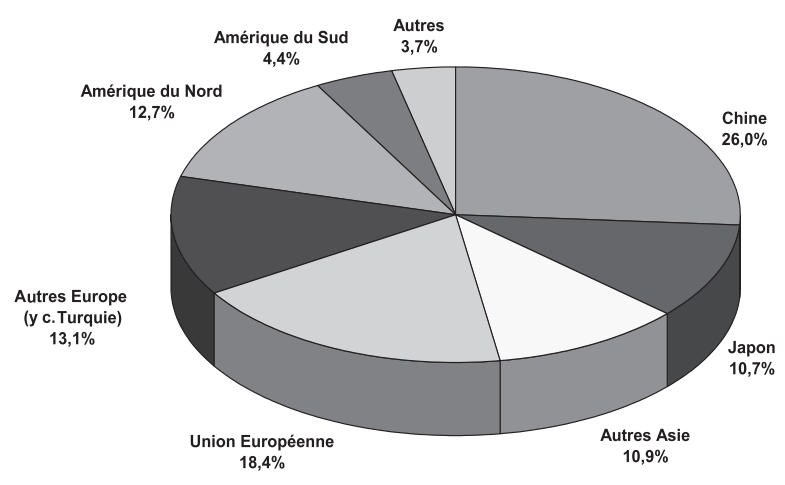

Production d'acier brut-Union Européenne à 25 Année 2004.

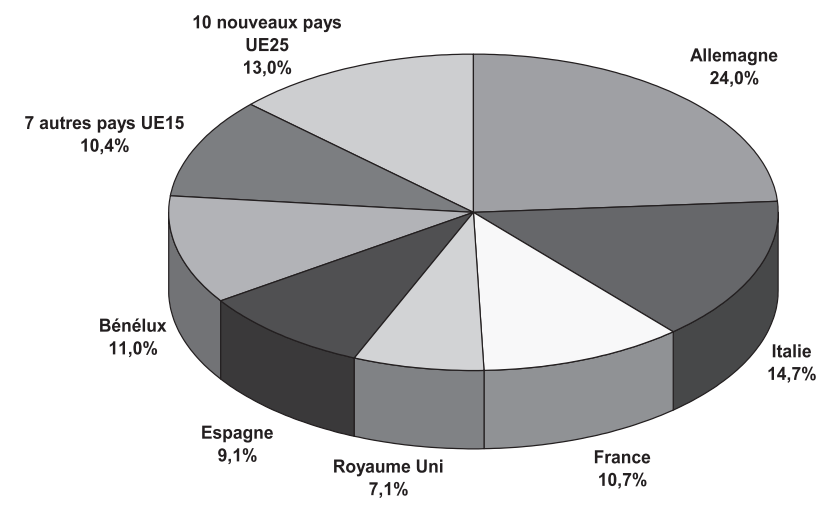

\section{Investissements - Recherche - Développement}

Les principaux investissements annoncés ou démarrés en 2003 concernent :

Le respect de l'environnement et les économies d'énergie

- GTS Industries a annoncé le lancement de la construction d'une installation de destruction des COV (composés organiques volatils).

- LME a procédé au démarrage d'une installation automatisée de capacité 7000 t/an pour l'enfournement de morceaux de pneus usagés au four électrique de Trith.

- SAM Montereau a construit un nouveau four de réchauffage des billettes équipé de brûleurs régénératifs.

- Sollac Atlantique, Dunkerque va procéder à la réfection de gazomètres et au démarrage début 2005 d'une nouvelle centrale à cycle combiné.

- Sollac Lorraine a procédé à la réfection de gazomètres.

- Sollac Méditerranée Fos a procédé à l'installation d'un dépoussiérage pour le décrassage de la fonte.

- Ugine \& ALZ Gueugnon a réalisé un prototype compact de chauffage par induction sur le recuit brillant. 
La satisfaction des besoins des clients et l'amélioration de la qualité des produits

- Ascometal Allevard a augmenté la section de la coulée continue (140 x 140)

- GTS Industries va installer 2 grosses couveuses pour la diffusion de l'hydrogène dans les tôles épaisses.

- SAM Neuves Maisons a mis en service une nouvelle coulée continue qui a atteint un niveau de fonctionnement très satisfaisant.

- Sollac Atlantique Dunkerque va transformer une coulée continue à et la doter d'une partie verticale pour faciliter la coulée des aciers pour l'industrie automobile et modifier le circuit d'évacuation des coils au train à bandes.

- Sollac Atlantique Mardyck va procéder à la transformation de sa ligne d'électrozingage en ligne de galvanisation ; le démarrage est prévu en avril 2005, après seulement 12 mois de travaux.

- Sollac Lorraine Florange élargit sa ligne d'étamage pour permettre la fabrication du fer blanc pour boîte boisson.

L'amélioration des coûts et les augmentations de capacité

- Alpa a procédé au revamping de la soudeuse de billettes du train à barres qui a atteint ainsi un taux de réussite d'environ $80 \%$.

- LME a installé des oxybrûleurs sur le four du train T320 lui permettant d'augmenter la capacité de production de 10 à $15 \%$ dans les périodes de marche saturée.

- Mittal Steel Gandrange reprend un rythme normal d'investissements destinés à l'amélioration de la fiabilité et de la productivité de l'aciérie et à l'augmentation de la capacité de parachèvement des deux trains à barres après deux années difficiles.

- Sollac Méditerranée Fos va construire une nouvelle batterie de 18 fours à coke.

- STUL a transféré la production du train de Longwy sur celui de Belval en mars 2005.

- Ugine \& ALZ a arrêté l'aciérie de L'Ardoise fin juin 2004.

Evolution des procédés et le développement de nouveaux produits

- Arcelor va démarrer en 2005 une ligne prototype industrielle de revêtement sous vide ; avecThyssen, Corus, Riva et de nombreux autres partenaires, il s'est engagé dans le projet européen ULCOS de réduction des émisions de $\mathrm{CO}_{2}$ de la sidérurgie ; dans le secteur des aciers inoxydables, il a développé de nouveaux aciers ferritiques pour l'industrie automobile ; dans le secteur des aciers plats carbone, il a poursuivi le développement des aciers à très haute résistance pour l'automobile et lancé la tôle sandwich Quiet Steel ; il procèdera au lancement d'une nouvelle métallurgie des aciers à haute teneur en manganèse en 2006 en association avec Thyssen et pourra grâce au projet ABC (Arcelor Body concept) offrir aux constructeurs automobiles de nouvelles solutions pour l'allègement et la sécurité des véhicules. Enfin, grâce à l'industrialisation de la technique de "slag splashing", il a pu sensiblement améliorer la durée de vie des convertisseurs de Fos.

- Ascometal met à disposition des constructeurs d'automobiles de nouvelles solutions aciers pour leurs nouveaux moteurs à puissance spécifique élevée (les aciers METAS$\mathrm{CO}$ pour les vilebrequins et SPLITASCO pour les bielles) et progresse dans la définition de nouvelles nuances résistant à la corrosion pour les ressorts automobiles dans le cadre de l'alliance technique avec KOBE.

- GTS Industries développe de nouveaux produits pour l'offshore et industrialise l'X80.

\section{Environnement}

Le Plan d'affectation des quotas a mobilisé la sidérurgie française tout au long de l'année 2004 ; les négociations avec l'Administration française ont été très serrées, mais elles se sont toutefois déroulées dans un climat d'écoute réciproque.

Les aciéries françaises ont obtenu un quota de 28,7 Mt/an de $\mathrm{CO}_{2}$ pour la période 2005/2007 ; ce challenge est atteignable, compte tenu des efforts antérieurement prévus par Arcelor dans le cadre de l'Association des Entreprises pour la Réduction de l'Effet de Serre (AERES).

Si l'on prend pour référence 1990 (référence du protocole de Kyoto), le Plan implique une réduction en valeur absolue de $6,6 \%$, pour des prévisions de production en hausse de $17,3 \%$; le gain sera donc de $20 \%$ en terme d'efficacité énergétique.

En matière de pollution de l'air, des investissements importants ont été engagés, à l'instar des années précédentes ; on peut citer le dépoussiérage de l'Aciérie de Montereau, et l'engagement de la première tranche de filtration additionnelle de l'usine d'agglomération du minerai de fer de Fos ; cette installation, la troisième en Europe mais la plus forte en capacité, est destinée à abattre drastiquement certains polluants, dont les dioxines. Elle devrait démarrer à mi-2005.

La pollution de l'air reste ainsi le domaine prioritaire des actions de progrès en cours, en particulier l'amélioration des connaissances des pollutions émises par les sites de production ; une compilation des méthodes d'estimation et des bases de données a permis, en liaison avec le Ministère de l'Environnement, la rédaction d'un guide méthodologique d'estimation des émissions ; cette action sera poursuivie, en particulier pour positionner les performances actuelles par rapport aux objectifs qui ont été fixés dans le cadre du Plan National Santé Environnement.

La préservation des ressources est également un souci partagé, notamment par l'utilisation de laitier sidérurgique en substitution de granulats naturels ; sur ce sujet, le Centre de Promotion Technique des Laitiers (CTPL) a lancé différentes actions techniques de caractérisation de produits, en liaison avec les administrations concernées.

Dans le secteur du bâtiment, la filière inox s'est occupée durant l'année 2004 de promouvoir de nouvelles solutions avec l'optique de "construire pour durer" en pensant à l'environnement (démarches HQE) et ce, bien entendu au moindre 
coût. Parmi celles-ci, les armatures inox pour béton qui offrent une solution satisfaisante quand il s'agit d'ouvrages sensibles aux dégradations dans les zones très exposées aux agents extérieurs tels les chlorures. Ce type d'applications nouvelles qui respecte à la fois les obligations économiques et écologiques, s'inscrit donc dans les critères du développement durable.

Malgré ces progrès constants, la sidérurgie française reste perplexe devant l'explosion réglementaire qui déferle sur elle depuis plusieurs années, et qui semble devoir s'amplifier encore.

\section{Recyclage}

Entre 2003 et 2004, le taux de consommation de ferrailles pour produire 1 tonne d'acier brut est passé de $51,4 \%$ à $51 \%$, diminution due au fait que la production par la filière électrique est plus faible en 2004.

Pour les produits en fin de vie, on distingue 4 marchés :

\begin{tabular}{|l|c|}
\hline Marchés & Taux de recyclage de l'acier \\
\hline Emballage & $62 \%$ \\
Electroménager & $75 \%$ \\
Automobile & $95 \%$ \\
Bâtiment & $75 \%$ \\
\hline
\end{tabular}

Le taux global de recyclage se situe entre 75 et $80 \%$.

\section{Normalisation}

Du fait de retards pris dans la procédure d'élaboration des normes européennes dans le secteur de l'acier, le nombre de normes françaises publiées en 2004 (29 normes) s'est situé, à un niveau bas, non constaté depuis 2001. A l'exception d'une seule de ces normes, celles-ci proviennent des filières européenne et internationale.
Le nombre de normes européennes (préparées ou instruites par l'ECISS - European Committee for Iron and Steel Standardization), approuvées dans le domaine des produits sidérurgiques et de la première transformation de l'acier, s'élève à 351 à la fin de l'année 2004. A cette date, le nombre de sujets inscrits au programme de travail de l'ECISS s'élève à 153 (- 7 par rapport à fin 2003), dont 75 études nouvelles (soit $49 \%$ du total).

2004 s'inscrit dans la continuité de 2003 avec le même nombre total de réunions tenues par les instances de normalisation internationale et européenne, suivies par les ingénieurs du BN Acier. Par contre, au niveau national, on relève une augmentation significative du nombre de réunions des instances de normalisation françaises.

Les autres réunions correspondent à des activités connexes à la normalisation : participation à des instances de certification de produits (AFNOR, AFCAB, ASQPE), à des instances d'accréditation (COFRAC), à des instances professionnelles (AIMCC, CEPMC, EUROFER) et à des instances chargées de réglementation (DGCCRF pour le contact alimentaire, DG santé pour le contact avec l'eau potable, groupe CWFT de la Commission européenne pour la réaction au feu des produits en acier de couverture et de bardage), au sein desquelles les ingénieurs du BN Acier sont amenés à représenter la profession de l'acier.

\begin{tabular}{|c|c|c|c|c|c|}
\hline \multicolumn{6}{|c|}{ Normes françaises publiées au cours des 10 dernières années dans le secteur de l'acier } \\
\hline \multirow{2}{*}{ Année } & \multicolumn{2}{|c|}{ Normes d'origine française } & \multicolumn{2}{|c|}{ Normes d'origine européenne } & \multirow{2}{*}{ Total } \\
\hline & nouvelles & révisions & nouvelles & révisions & \\
\hline 1995 & 5 & - & 29 & - & 34 \\
\hline 1996 & 6 & 4 & 22 & 3 & 35 \\
\hline 1997 & 4 & - & 14 & 4 & 22 \\
\hline 1998 & 3 & 1 & 25 & 2 & 31 \\
\hline 1999 & - & 5 & 35 & 9 & 49 \\
\hline 2000 & 3 & - & 40 & 3 & 46 \\
\hline 2001 & 3 & 3 & 20 & 2 & 28 \\
\hline 2002 & 9 & 3 & 34 & 5 & 51 \\
\hline 2003 & 7 & 1 & 32 & 10 & 50 \\
\hline 2004 & 1 & 0 & 14 & 14 & 29 \\
\hline
\end{tabular}

